\title{
Platon et la Paideia : l'horizon démocratique
}

Plato and Paideia: The democratic horizon

Platón y la Paideia: el horizonte democrático

Didier Moreau

\section{OpenEdition}

\section{Journals}

Édition électronique

URL : https://journals.openedition.org/ries/7018

DOI : 10.4000/ries.7018

ISSN : 2261-4265

Éditeur

France Education international

Édition imprimée

Date de publication : 1 décembre 2018

Pagination : 53-60

ISBN : 978-2-85420-620-3

ISSN : $1254-4590$

Référence électronique

Didier Moreau, «Platon et la Paideia : l'horizon démocratique », Revue internationale d'éducation de Sèvres [En ligne], 79 | décembre 2018, mis en ligne le 01 décembre 2020, consulté le 24 juin 2021 URL : http://journals.openedition.org/ries/7018 ; DOI : https://doi.org/10.4000/ries.7018 


\title{
Platon et la Paideia : l'horizon démocratique
}

\author{
Didier Moreau \\ Université Paris 8 Vincennes-Saint-Denis
}

Parmi les concepts qui structurent le champ éducatif, en orientent les pratiques et en organisent les catégories de pensée, celui de Paideia détient une importance fondamentale, liée à sa position originelle. Comme tous les concepts inauguraux en philosophie, il conserve son noyau métaphorique qui le rattache aux jeux et à l'enfance et se plaît à le faire valoir. Werner Jaeger (1964), dans l'avertissement qui ouvre son ouvrage éponyme, note que ce concept ne peut plus être saisi comme tel par la pensée moderne qui ne peut l'approcher que dans le foisonnement de notions éclatées comme : « civilisation, tradition, littérature ou éducation. (...) En les prenant toutes ensemble, on ne saurait les employer pour exprimer le sens complet du mot grec. »C'est ce qui l'autorise aussitôt, par une identification conceptuelle étonnante, à traduire Paideia par Bildung, ce qui ne laisse plus d'autre choix au traducteur français que de le restituer par culture ${ }^{1}$, opération qui a été incorporée par l'usage.

Ce n'est donc pas cette complexité de la structure du concept que nous aborderons ici, un tel travail nécessiterait un commentaire critique de la thèse centrale de Jaeger, mais plutôt voudrions-nous tenter de comprendre, à travers ce thème de la rupture entre le monde grec et notre modernité, comment la Paideia participe toujours de notre horizon éducatif et, disons-le avec Jaeger, "civilisationnel ». Notre hypothèse est ici que le concept est par lui-même à l'origine de la rupture : les temps modernes, comme époque historique, au sens de Blumenberg (1999), sont une conséquence directe de l'orientation de la pensée de l'humanisme ancien qu'elle fonda.

\section{la Paideia et la Polis}

Il y a paradoxalement peu de textes des philosophes grecs anciens qui permettraient de saisir le concept en compréhension. Plusieurs interprétations sont possibles, la plus générale étant qu'il fait partie du sol commun de la réflexion philosophique sur l'éducation et qu'il faut plutôt le défendre contre l'extériorité d'une pensée qui voudrait se faire valoir par fraude comme philosophique. Ainsi, la Paideia se trouverait constituée à la fois comme moyen et fin pour la philosophie, moyen pour y parvenir, l'éducation de soi-même se trouvant promue, du même coup, comme fin de l'activité philosophique. C'est la thèse majeure de Pierre Hadot, pour qui, depuis la Grèce archaïque, la Paideia a nourri toute pratique éducative socialement organisée.

1. W. Jaeger, Paidea, Die Formung des griechisches Menschen, Berlin, Walter de Gruyter, 1936. La traduction en français, revue par l'auteur, est de André et Simonne Devyver. 
Platon évoque, dans un passage des Lois (1950), l'importance d'une éducation de la jeunesse dans la constitution de la Cité. Du point de vue d'une construction philosophique, une telle éducation doit permettre à chaque citoyen de distinguer le juste et l'injuste, non seulement par l'intellection, mais de surcroît de manière affective,

[afin que] du commencement à la fin de l'existence, on haïsse ce qu'il faut haïr et qu'on chérisse ce qu'il faut chérir, voilà ce que j’appelle justement " éducation " (Paideia).

Tous les éléments constitutifs sont ici présents, l'horizon téléologique, qui ne dissocie pas l'éducation individuelle de la constitution d'une Cité juste, et l'appel à la totalité des parties de l'âme, qui doivent concourir de manière réglée à ce que le sujet s'engage résolument dans l'action droite. Cette définition permet d'écarter à rebours les "fausses » éducations, qui vont s'appuyer sur des représentations trompeuses et des sentiments artificiellement suscités. Or les seules pratiques qui, pour Platon, garantissent la régulation circulaire des mouvements du corps et de l'âme sont les danses, selon une musique elle-même harmonieuse : il y a le même rapport dans l'individu entre les parties de son corps et celles de son âme que dans un groupe de danseurs où chacun exécute ses mouvements en harmonie (sans qu'il soit nécessaire de les rendre tous uniformes) avec ceux des autres danseurs. Cette perspective est essentielle pour saisir le concept de Paideia. Il s'agit d'apprendre à se régler et à s'orienter vers l'horizon commun d'un être-ensemble harmonieux que réalisera la Cité, en aucun cas, de retrancher une partie de soi, de renoncer à ses affects et encore moins à son aspiration au plaisir. Le moyen utilisé ne peut pas être contraignant car une contrainte introduirait la crainte et la sujétion. Ce sera - ce qui contredit toutes les perspectives autoritaristes - le partage d'un plaisir commun, seul apte à conduire sur la voie d'une expérience mixte où se mêlent, à égalité, les plaisirs individuels et les peines.

[E]n conséquence, celui qui a reçu une belle éducation doit sans doute être capable de chanter comme de danser de la belle manière. ${ }^{2}$

Mais comment conduire à un tel résultat ? Comment s'initie la Paideia ? Le Livre VII des Lois, complexe et que l'on commente peu car on le juge peu conforme à une doxa éducative reçue du platonisme, ouvre des perspectives étonnamment éclairantes pour nous, contemporains qui réfléchissons sur l'éducation au sein d'un êtreen-commun, en vue de son renforcement. L'axiome inaugural de toute Paideia est " qu'on a affaire à des sujets de condition libre ${ }^{3}$. La Paideia d'un sujet libre ne peut donc être initiée que par ce sujet même ; c'est le paradoxe de toute éducation libre, qui ne sera contourné que par les métaphysiques pessimistes d'Augustin, Kant ou Schopenhauer, Durkheim et Levinas, pour lesquelles seule l'exercice d'une contrainte et d'une violence permet d'apporter une correction au penchant pour le mal, représenté par le péché originel, le mal radical, la sauvagerie asociale ou l'altérité radicale. La Paideia est ainsi la clef qui ouvre sur les éducations confiantes et les formations de soi mutualisées. L'on y retrouve le noyau métaphorique originel, entre le jeu et l'enfance, que Platon articule dialectiquement:

2. ibid. 654 a, p. 674 .

3. ibid. 794 , a, p. 869 . 
[...] chez des êtres d'un tel âge [les enfants], il existe des jeux naturels et spontanés, qu'ils ne sont pas loin de découvrir d'eux-mêmes aussitôt qu'ils se trouvent réunis. Or c'est sur les terrains consacrés de chaque village que devront dorénavant se réunir tous les enfants qui ont l'âge en question, c'est-à-dire de trois à six ans ${ }^{4}$.

Tous les enfants, garçons et filles, enfants de citoyens, d'esclaves et d'étrangers, sont ainsi rassemblés, sous la surveillance de préposées désignées par les Gardiens-des-Lois, dans un topos commun, "école ouverte » en quelque sorte, et qui n'ont d'autres prérogatives que de veiller à leur sécurité et à la décence vis-à-vis de lois de la Cité. Qu'y font-ils ? Ils jouent à être ensemble, non pas à se socialiser, idée étrangère à la pensée antique, mais à construire les lois qui soudent une communauté. ${ }^{5}$ L'instruction viendra ensuite qui orientera chacun, à partir de ses dispositions personnelles, vers la contribution qu'il peut apporter à la Cité. Ce topos, antérieur à la skolé, est ce qui la rend possible ; l'ignorer, c'est manquer la Paideia et, disons-le, manquer de Paideia soi-même. Tous les éducateurs qui s'en inspireront, pour ne prendre en considération que les Temps modernes, Comenius, Rousseau, Basedow, Pestalozzi, Hermann Lietz, Ferrière, Piaget, Paulsen, Cousinet, Freinet et bien d'autres, poseront cette double axiomatique croisée au cour de leur doctrine pédagogique, en articulant les apprentissages et le jeu libre, la première enfance et la mutualisation. Le texte des Lois ne se prive pas, au demeurant, de jouer sur les mots : Païdeïa et Païdia, éducation et jeu ${ }^{6}$.

Mais cette initiation, qui se nourrit de la spontanéité enfantine, est organisée autour du souci d'un être-en-commun dont l'idéal éducatif est la source. C'est le problème philosophique par excellence, celui de construire une tradition qui ne soit pas répétition du même et du déjà-donné, mais qui dégage un réel horizon de perfectionnement moral de l'être-ensemble, de la Polis, ou à défaut, qui la protège de la corruption du temps qui passe et de l'usure des habitudes. Le texte des Lois pose remarquablement cette aporie, à travers la figure du législateur mythique.

Mais un législateur [...] n'aurait-il pas eu la hardiesse [...] d'inventer pour le bien, en s'adressant à la jeunesse, une fiction mensongère ?

L'objectif est d'obtenir une plus grande efficacité « pour que tous, non par la contrainte mais de leur plein gré, ils aient une conduite plus juste. ${ }^{8}$ La fiction mensongère, pour prix de la liberté, en quelque sorte... Mais de quelle fiction mensongère s'agit-il ? C'est une nouvelle aporie dont on doit s'extraire, reprise de celle à laquelle répondait le Ménon : on ne peut pas apprendre car soit l'on sait, soit l'on ne sait pas ; celui qui sait ne peut plus apprendre et celui qui ne sait pas ignore quel objet rechercher. Le législateur mythique la reformule ainsi : comment transmettre ce que l'on tient pour vrai, qui est l'idéal de l'être-en-commun, et qui, du point de vue de la communauté, sera plus juste que le repli sur soi ou sur son clan familial ? Comment faire croire à la vérité ? Cette question reste la nôtre et l'on se demande toujours ce que signifie croire en la vérité, à l'ère de ce que l'on nomme

\footnotetext{
4. ibid., p. $869-870$.

5. Aristote ne dit pas autre chose : «La cité est une pluralité qui, par le moyen de l'éducation, doit être ramenée à une communauté et à une unité. » Politique, II, 5, 1263 b, Paris, Vrin, 1977, p. 36-37.

6. Platon, ibid. 656 c, p. 678.

7. ibid. 663 e, p. 689.

8. ibid.
} 
la " post-vérité ", ère pour laquelle le problème est que la falsification - et les théories du complot qui en résultent - est plus attirante que la vérité. Ce problème n'est pas sociologique mais philosophique ; il est retrouvé par Wittgenstein dans la théorisation des «formes de vie » qui donnent confiance dans des propositions indémontrables ${ }^{9}$; n'est-ce pas là l'horizon de la Paideia ? Constituer une communauté, hors de toute vérité métaphysiquement transcendante, qui ne repose que sur son propre horizon invérifiable : "Vérifier, cela n'a-t-il pas un terme ? ", demande Wittgenstein (1965).

Certes, on peut concevoir que les grands récits participent de cette mise en fiction, à cette différence près qu'ils ne laissaient aucun espace de jeu de recomposition, de réagencement et de réécriture. La communauté de la Paideia n'est pas fondée sur la nostalgie d'une origine sacralisée, dont le culte en serait, par essence, antinomique. La répétition de l'origine ne tolère aucun écart compromettant la pureté de ce qu'elle remémore. C'est pourquoi la fiction mensongère des Lois n'a d'autre horizon que le rassemblement dans le plaisir d'être ensemble :

Par conséquent, le législateur n'a besoin de chercher à découvrir rien d'autre que de savoir quelle croyance, donnée par lui à la Cité, réaliserait pour elle le plus grand bien et [donc] à découvrir, à cet égard, [...] de savoir de quelle manière, là-dessus, cette communauté tout entière parlera toujours, durant la totalité de son existence, le plus possible d'une seule et même voix, aussi bien dans ses chants que dans ses légendes ou dans ses propos. ${ }^{10}$

Le texte organise alors un système de trois chœurs, selon l'âge des citoyens, par lesquels se transmet le mythe éducatif : les plus âgés attestant pour les plus jeunes du pouvoir de formation de la Paideia reçue du point de vue des « dispositions morales ${ }^{11}$. Ce dispositif complexe est la trame de la culture comme éducation et de l'éducation comme culture. C'est la clef de l'identification, que Jaeger reprend à son compte, comme on l'a vu, entre Bildung et Paideia. Les moyens en sont techniques et les buts éthiques : les arts, comme structures de régulation des mouvements de l'âme, préfiguration de l'Esprit objectif de Hegel, pour qui les réalisations visibles de la culture ont une fonction d'éducation de la subjectivité.

La tradition éducative jusqu'à nous se nourrit de cette perspective éthico-ludique, pourrait-on dire, de la Paideia. On la trouve réaffirmée avec force par Rousseau, dans ses Considérations sur le gouvernement de Pologne, et thématisée comme horizon collectif de la fête, dans la Lettre à d'Alembert sur les spectacles, dans laquelle au demeurant est liquidée l'hypothèque - insupportable dans le système de véridicité rousseauiste - d'une fiction mensongère : la fête populaire, comme autofondatrice, est autosuffisante pour l'établissement d'une vie éthique collective. Jules Michelet (1939), à la suite de Rousseau, verra dans les fêtes des fédérations un moment d'ontologie politique, et Schleiermacher (2004) théorisera, à partir d'une intuition herméneutique de premier plan, la fête comme horizon de la Bildung, où fusionnent l'universel et le singulier.

9. Mais Wittgenstein donne aussi des exemples qui nous interpellent : on ne peut pas douter que nous soyons nés de parents ou qu'aucun homme n'a marché sur la Lune. La confiance, comme l'amitié, ne se fondent pas, ce sont des actes de donation originaires.

10. Platon, Les Lois, 664 -a, p. 691.

11. ibid. 


\section{Paideia et démocratie}

Il y a une longue histoire de la réception du concept de Paideia, dans la pensée moderne, qui alimente, ponctue et oriente de manière décisive les théories politiques et éducatives. Cette histoire possède, malgré une diversité des interprétations et des systèmes philosophiques concernés, une unité de ton tout à fait remarquable. Cette unité consiste dans la référence à ce que l'on nomme l'humanisme ancien, qui est un ensemble axiologique centré sur la possibilité d'une formation de soi-même obtenue par une appropriation collective de la culture partagée. Cette référence est un effort pour faire "renaitre " (Rinascimento, Renaissance), ce que l'antihumanisme augustinien avait condamné : la culture comme affirmation positive de la place de l'homme dans un cosmos dont il devait prendre connaissance pour se perfectionner moralement (Marrou, 1958). Cet effort de réappropriation, en outrepassant ou contournant les dogmes de la théologie, que l'on trouve chez les auteurs de la Renaissance, et chez Montaigne principalement, se structure selon deux directions majeures : une critique précise et méticuleuse de ce qui constitue la base de la transmission culturelle (Jehasse, 2002) et une méditation approfondie sur la condition propre de la modernité (l' « humaine condition » chez Montaigne, indifférente désormais à la situation onto-théologique de l'homme comme créature divine). Toute illusion selon laquelle la religion pouvait rendre les hommes meilleurs étant alors abandonnée, les penseurs ne pouvaient plus compter que sur leurs propres forces pour se former. Le thème de l'autoformation de l'homme devient alors un thème central de la Renaissance érudite du Xvi ${ }^{e}$ siècle, dynamisé par cette déception partagée selon laquelle les doctrines des Anciens ne possédaient pas les réponses aux questions des temps nouveaux.

Ainsi, le travail d'érudition d'Henri Estienne et de ses contemporains, Juste Lipse, Joseph Scaliger et Isaac Casaubon, répond-il à une volonté manifeste de se former par une lecture critique des œuvres transmises et qui, ainsi, concourent à la Paideia de l'époque. La réappropriation de la Paideia acquiert alors une fonction critique double : dénoncer ce qui, dans les institutions politiques et sociales fait écran, ou même obstacle, au déploiement de la liberté humaine entendue comme devoir vis-à-vis de ce que l'on a reçu en héritage et ce que l'on doit transmettre, et ouvrir un espace utopique de ce que pourrait être un corps politique orienté radicalement vers le perfectionnement de soi à travers l'être collectif.

L'époque des Lumières reprend cette orientation générale. Diderot définit ainsi le projet de l'Encyclopédie :

Encyclopédie. Ce mot signifie enchaînement de connaissances; il est composé de la préposition grecque $\mathrm{ev}$, en, et des substantifs kuklov, cercle et Paideia, connaissance. En effet, le but d'une Encyclopédie est de rassembler les connaissances éparses sur la surface de la terre; d'en exposer le système général aux hommes avec qui nous vivons, et de le transmettre aux hommes qui viendront après nous ; afin que les travaux des siècles passés n'aient pas été des travaux inutiles pour les siècles qui succéderont; que nos neveux, devenant plus instruits, deviennent en même temps plus vertueux et plus heureux, et que nous ne mourions pas sans avoir bien mérité du genre humain.

Cet idéal est éthique et politique, qui associe la vertu et le bonheur, lorsque l'antihumanisme n'a de cesse de les séparer radicalement. 
Mais quelle est donc cette puissance originelle de la Paideia, qui la constitue, à travers les sociétés historiques héritières de l'humanisme ancien, comme l'horizon indépassable de toute éducation libre ? Michel Foucault (2001) note, dans son analyse de la modernité, que, structurellement, pour comprendre des ruptures qui semblent récentes, il faut remonter à une origine grecque ou hellénistique. Quel est donc le secret grec de la Paideia ? Werner Jaeger (op. cit.) nous livre une clef précieuse. Son livre, il faut s'en souvenir, paraît en octobre 1933, " en ces temps présents où nous combattons pour sauver notre civilisation millénaire ${ }^{12}$. Sa démarche s'appuie sur la théorie des trois humanismes : si la Renaissance a eu pour horizon de refonder la dynamique de l'humanisme ancien, contre la théologie, est-il possible de puiser, dans l'Antiquité classique, une " autorité éducative " susceptible de devenir la source d'une nouvelle formation humaniste, la troisième donc ? Son ouvrage est une réponse négative. Notre époque a définitivement «pulvérisé » l'idéal grec et les humanismes modernes ne sont que des euphémisations de l'individualisme contemporain. Abandonnant alors la perspective d'une restauration qui serait suspecte politiquement, Jaeger s'attache à cerner le concept de Paideia, au cœur de la culture grecque. Ce livre magistral est à l'origine de réorientations décisives de la philosophie de l'éducation contemporaine. Ainsi, Pierre Hadot (1995) s'y réfère-t-il centralement pour saisir le concept de Paideia, en pointant la différence entre éducation reproductrice d'une classe sociale et culture. Mais c'est bien cette lecture jaegerienne qui guidera Hadot vers l'étude de la philosophie comme formation pratique de soi, source reconnue de l'herméneutique du sujet de Foucault.

Quel est donc ce "secret» de la Paideia ? La structure majeure du projet éducatif est énoncée dans une note de l'édition de 1936 : en demandant quelle est la raison majeure du rayonnement universel de la pensée grecque, il met en exergue l'élément de rationalité de la culture qui la pousse à se développer

sans se soucier des barrières de classes au point de devenir la culture de la nation hellénique tout entière et, en définitive, la civilisation d'un monde. (ibid. note 6 , p. 475.)

Comme mode de formation, l'éducation humaniste serait ainsi un mode de subjectivation qui n'attribue pas de " places » et qui ouvre l'espace dans lequel les mots peuvent s'échanger hors de tout rang pré-désigné : son principal résultat historique, analyse-t-il dans la même note, est alors la «métamorphose de l'antique style de vie aristocratique en la démocratie du temps de Périclès. ${ }^{13}$

Cette inversion est décisive et Hadot reprend cette transformation d'une éducation en vue de l'arété, de l'excellence aristocratique, en une formation du citoyen de la Cité démocratique. Insistons sur cette interprétation de Jaeger. La Paideia est une projection de la Cité dans son propre perfectionnement historique, qui se perçoit et se comprend dans l'excellence de chacun pris indifféremment. Cet idéal est la formation d'une universalité rationnelle qui transcende, d'un point de vue non métaphysique, les particularités historico-sociales qui fragmentent la Cité dans des conflits d'intérêts privés. Le « bon législateur » est celui qui, par la fiction

12. Jaeger attendra que son discours pour le tricentenaire de l'Université de Harvard en 1936 soit censuré par le ministre du Reich pour quitter sa chaire de Berlin.

13. ibid. p. 474 . 
originaire, oriente la Cité dans un même élan pour la faire parler d'une même voix ». Apparaît alors pour Jaeger la coappartenance de la Paideia et de la démocratie : l'une et l'autre sont inextricables. Le but de la Paideia est de réaliser la démocratie, le moyen et le milieu ambiant de la démocratie sont de développer la Paideia. Le milieu de culture est celui de la connaissance rationnelle, qui pousse le législateur mythique à préparer son extension. Ainsi Solon écarte-t-il le risque de la fragmentation " individualiste », en dotant chacun d'une personnalité par le perfectionnement de soi, laquelle permet au premier législateur de se retirer pour que chacun - et tous ensemble - puissent poursuivre sa tâche. Foucault se réfère, en quelques occasions, à cette formule de Jaeger, sans la citer :

[L] a qualité très rare [pour établir des lois], Solon l'appelle gnomosyné, ou discernement, parce qu'en toute circonstance, elle suscite la gnomé - c'est-à-dire à la fois une connaissance réelle et la volonté de lui donner une application pratique. ${ }^{14}$

En faisant apparaître la Paideia comme la source de l'élan démocratique, Jaeger (op. cit.) introduit un déplacement, peu visible dans la traduction française, par lequel il substitue Formung à Bildung et fait de l'éducation une véritable formation du sujet. Cette substitution est explicitée par l'opposition entre les « choses muettes » et la parole vivante :

Pourtant les Grecs crurent que les véritables représentants de la Paideia étaient non pas ceux qui font profession des choses muettes (sculpteurs, peintres, architectes), mais les poètes, les musiciens, les orateurs [les hommes d'État], ainsi que les philosophes.

Cette substitution autorise un mode de subjectivation qui se réalise lorsque le poète opère comme un sculpteur, un formateur au sens propre, agissant sur du vivant :

[I]ls sentirent en effet que le législateur était (dans un certain sens) plus proche du poète que ne l'était le sculpteur car tous deux, poète et législateur, assumaient une vision éducative. Seul le législateur pouvait prétendre au titre de sculpteur, car seul il modelait l'homme vivant. (ibid.)

Si on ne forme un homme que "par les mots et les sons ", la Cité ne peut être qu'un espace de partage de la parole où chacun, comme dans les trois chœurs des Lois, peut faire entendre sa voix, dans ce milieu où circule ce que Jaeger appelle l' "énergie active» (ibid.). Le terme de Bildung devient alors trop faible - ou affaibli par les institutions contemporaines - pour nous rappeler son sens originel ; mais cet affaiblissement était inévitable, car il trouve sa racine dans l'équivoque de la métaphore platonicienne, qui insiste sur le travail du sculpteur, alors qu'elle recherche, dans les Lois, comment former par la parole des citoyens orientés vers la justice. Mais on se souvient aussi que Platon ne préconise pas la démocratie...

Il nous semble alors que cette équivoque reste la nôtre, lorsque nous pensons l'éducation plutôt comme une distribution (inégalitaire) de biens que comme une véritable formation collective prise en charge par une culture vivante. Pour conclure, penser en direction de la Paideia, dans la perspective la plus modeste - et donc la plus libre possible - rejoindrait ce souci formulé par Aristote (1969) : 
Enseignant non pas l'art, mais les résultats de l'art, ils [les sophistes] s'imaginaient qu'en cela consistait l'éducation : c'est comme si, prétendant transmettre la science de n'avoir pas mal aux pieds, on enseignait à quelqu'un non pas l'art de faire des chaussures ni même à savoir se procurer des choses de ce genre, mais on se bornait à lui présenter plusieurs espèces de chaussures de toutes sortes; ce serait là lui donner un secours pratique, mais non pas lui transmettre un art.

Sans chaussures adaptées, il n'est pas possible de parcourir le monde, de se rendre aux réunions publiques ni de participer aux fêtes collectives, en un mot de concourir à renforcer la vie démocratique.

\section{RÉFÉRENCES BIBLIOGRAPHIQUES}

ARISTOTE (1969) : Les réfutations sophistiques, 184 a, b, Paris, Vrin.

BLUMENBERG H. (1999) : La légitimité des Temps modernes, Paris, Gallimard.

DIDEROT D., (1757) : "Encyclopédie », dans Diderot et d'Alembert, Encyclopédie ou Dictionnaire raisonné des sciences, des arts et des métiers.

FOUCAULT M. (2001) : Dits et écrits, t. II, Paris, Gallimard.

HADOT P. (1995) : Qu'est-ce que la philosophie antique?, Paris, Gallimard.

JAEGER W. (1964) : Paideia, la formation de l'homme grec, Paris, Gallimard.

JEHASSE J. (2002) : La renaissance de la critique, Paris, Honoré Champion.

PLATON (1950) : Les Lois, II, 653 c, Euvres complètes, t.2, Paris, La Pléiade.

SCHLEIERMACHER F. D. (2004) : Esthétique, tous les hommes sont des artistes, Paris, Cerf.

WITTGENSTEIN L. (1965) : De la certitude, Paris, Gallimard. 